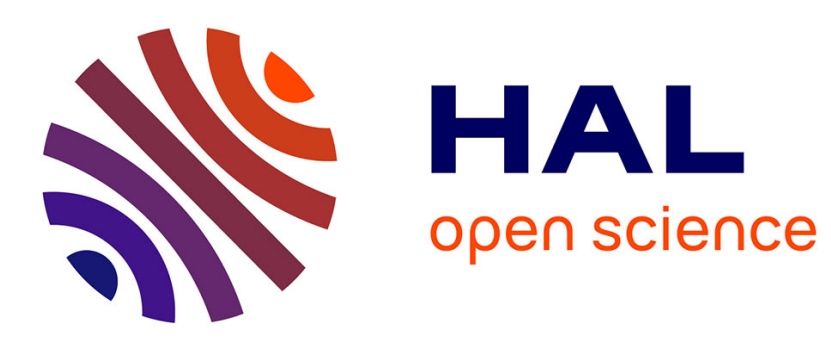

\title{
On a new class of 2-orthogonal polynomials, I : The recurrence relations and some properties
}

\author{
Khalfa Douak, Pascal Maroni
}

\section{To cite this version:}

Khalfa Douak, Pascal Maroni. On a new class of 2-orthogonal polynomials, I: The recurrence relations and some properties. Integral Transforms and Special Functions, In press, 10.1080/10652469.2020.1811702 . hal-02958834

\section{HAL Id: hal-02958834 \\ https://hal.sorbonne-universite.fr/hal-02958834}

Submitted on 6 Oct 2020

HAL is a multi-disciplinary open access archive for the deposit and dissemination of scientific research documents, whether they are published or not. The documents may come from teaching and research institutions in France or abroad, or from public or private research centers.
L'archive ouverte pluridisciplinaire HAL, est destinée au dépôt et à la diffusion de documents scientifiques de niveau recherche, publiés ou non, émanant des établissements d'enseignement et de recherche français ou étrangers, des laboratoires publics ou privés. 


\title{
On a new class of 2-orthogonal polynomials, I : The recurrence relations and some properties
}

\author{
Khalfa DOUAK* and Pascal MARONI ${ }^{\dagger}$ \\ Laboratoire Jacques-Louis Lions, Université Pierre et Marie Curie-CNRS, \\ Boite courrier 187, 75252 Paris Cedex 05, France \\ Email: *khalfa.douak@gmail.com and †maroni@ljll.math.upmc.fr
}

\begin{abstract}
The classical 2-orthogonal polynomials share the so-called Hahn property, this means that they are 2-orthogonal polynomials whose the sequences of their derivatives are also 2-orthogonal polynomials. Based only on this property, a new class of classical 2-orthogonal polynomials is obtained as particular solution of the non-linear system governing the coefficients involved in the recurrence relation fulfilled by these polynomials. A differential-recurrence relation as well as a third-order differential equation satisfied by the resulting polynomials are given. Many interesting subcases are highlighted and explicitly presented with special reference to some connected results that exist in the literature. The integral representations of their associated linear functionals will be exhaustively discussed in a forthcoming publication.
\end{abstract}

Keywords. Orthogonal polynomials, classical orthogonal polynomials, d-orthogonal polynomials, multiple orthogonal polynomials, recurrence relation, differential equations.

AMS Classification. 33C45; 42C05.

\section{Introduction}

The $d$-orthogonal polynomials are polynomials of one variable satisfying a specific orthogonality relations with respect to (w.r.t.) $d$ different linear functionals $[1,2]$. This concept is a naturel extension of the general orthogonal polynomials relative to a regular linear functional. By means of this notion, the second author showed that, the $d$-orthogonality of a given sequence of polynomials w.r.t. any vector of $d$ linear functionals is equivalent to the $d$-orthogonality of these polynomials relative to the vector consisting of the first $d$ elements of its associated dual sequence, see [1, Th. 2.1]. That is why, instead of considering the orthogonality conditions w.r.t. any set of linear functionals, it is somewhat easier and more naturel to consider directly the orthogonality conditions w.r.t. the first $d$ functionals of the dual sequence. It should be noted that there are many $d$-orthogonal polynomials for which the weight functions are not known or are not unique but has a characteristic high-order standard recurrence

\footnotetext{
${ }^{*}$ The corresponding author.
} 
relation. That is why, the questions of the integral representations of the associated linear functionals will be considered posteriorly wherever possible (here for $d=2$ ).

The paper is organized as follows. In the rest of this section we recall some known results on the ordinary orthogonality as well as on the $d$-orthogonality of polynomials which we need in the sequel. Section 2 is devoted to the introduction of the new class of 2-orthogonal polynomials with its explicit recurrence coefficients. In Section 3, we provide some properties satisfied by the resulting polynomials and to conclude we enumerate many interested subcases.

\subsection{The orthogonal polynomials}

First of all, let us recall some basic notations that we will use through this paper as introduced, for instance, in [3]. Let $\mathscr{P}$ be the vector space of polynomials of one variable with complex coefficients and $\mathscr{P}^{\prime}$ its algebraic dual. By $\langle.,$.$\rangle we denote the duality brackets between \mathscr{P}$ and $\mathscr{P}^{\prime}$. Let us denote by $\left\{P_{n}\right\}_{n \geqslant 0}$ a polynomials sequence (PS in short), with $\operatorname{deg} P_{n}=n$, and $\left\{u_{n}\right\}_{n \geqslant 0}$ its associated dual sequence defined by $\left\langle u_{n}, P_{m}\right\rangle=\delta_{n m} ; n, m \geqslant 0$, where $\delta_{n m}$ is the Kronecker's delta symbol. Throughout this article, we will always consider the sequence of monic polynomials, i.e. the leading coefficient of each polynomial $P_{n}$ is one $\left(P_{n}(x)=x^{n}+\cdots\right)$. If a PS $\left\{P_{n}\right\}_{n \geqslant 0}$ is not monic and $k_{n}$ denotes the leading coefficient of $P_{n}(x)$, then $\hat{P}_{n}(x)=k_{n}^{-1} P_{n}(x)$ yields the corresponding monic PS, $\left\{\hat{P}_{n}\right\}_{n \geqslant 0}$.

Given a linear functional $u \in \mathscr{P}^{\prime}$. The sequence of complex numbers $(u)_{n}, n=0,1,2, \ldots$, denotes the moments of $u$ with respect to the sequence $\left\{x^{n}\right\}_{n \geqslant 0}$, namely, the moment of order $n$ for the functional $u$ is denoted by $(u)_{n}:=\left\langle u, x^{n}\right\rangle$. Thus, the linear functional $u$ is completely determined by its moments. To terminate we define by $\Delta_{n}=\operatorname{det}\left((u)_{i+j}\right)_{i, j=0}^{n}, n \geqslant 0$, the ordinary Hankel determinants constructed from the moments of $u$ (see e.g. [3] and the references given there).

Definition 1.1 : A PS $\left\{P_{n}\right\}_{n \geqslant 0}$ is said to be orthogonal w.r.t. the linear functional $u$, if it satisfies the orthogonality conditions

$$
\begin{gathered}
\left\langle u, P_{n} P_{m}\right\rangle=0, n \neq m, \\
\left\langle u, P_{n}^{2}\right\rangle \neq 0, n \geqslant 0 .
\end{gathered}
$$

In this case (1.2) are said to be the regularity conditions.

Theorem 1.2 [4]: Let $u$ be a linear functional with moments sequence given by $(u)_{n}, n \geqslant 0$. A necessary and sufficient condition for the existence of an orthogonal polynomials sequence (OPS) $\left\{P_{n}\right\}_{n} \geqslant 0$ with respect to $u$ is $\Delta_{n} \neq 0$ for $n=0,1,2, \ldots$. The functional $u$ is then said to be regular.

Equivalently, the polynomials $P_{n}, n=0,1,2, \ldots$, are orthogonal if and only if they satisfy the well-known second-order recurrence relation

$$
P_{n+2}(x)=\left(x-\zeta_{n+1}\right) P_{n+1}(x)-\xi_{n+1} P_{n}(x), n \geqslant 0,
$$

with the regularity conditions $\xi_{n+1} \neq 0, n \geqslant 0$, and the initial conditions

$$
P_{0}(x)=1, P_{1}(x)=x-\zeta_{0} .
$$




\section{Remarks 1.1:}

(a) The coefficient $\xi_{0}$ does not appear in the recurrence relation (1.3), it is convenient here to set $\xi_{0}:=(u)_{0}$. When $\xi_{0}=1$, the linear functional $u$ is said to be normalized.

(b) In view of the orthogonality conditions (1.1)-(1.2), the coefficients $\zeta_{n}$ and $\xi_{n}$ are given by

$$
\zeta_{n}=\frac{\left\langle u, x P_{n}^{2}\right\rangle}{\left\langle u, P_{n}^{2}\right\rangle}, n \geqslant 0, \text { and } \xi_{n+1}=\frac{\left\langle u, P_{n+1}^{2}\right\rangle}{\left\langle u, P_{n}^{2}\right\rangle}, n \geqslant 0, \text { with }\left\langle u, P_{n}^{2}\right\rangle=\prod_{k=0}^{n} \xi_{k}, n \geqslant 0 .
$$

In addition, we have $\Delta_{n+1}=\Delta_{n}\left\langle u, P_{n+1}^{2}\right\rangle$, yielding $\xi_{n+1}=\Delta_{n-1} \Delta_{n+1} / \Delta_{n}^{2}, n \geqslant 0$, (where $\Delta_{-1}:=$ 1).

(c) It follows from (1.2), that if an OPS for a linear functional $u$ exists, then $(u)_{0} \neq 0$. Thus, no regular OPS can exist if $(u)_{0}=\langle u, 1\rangle=0$.

\subsection{The $d$-orthogonal polynomials}

It should firstly be noted that the $d$-orthogonal polynomials are considered as an interesting subclass of type II multiple orthogonal polynomials whose multi-indices lie on the step-line near the diagonal ${ }^{1}$. In the literature, most of the people working on the multiple orthogonal polynomials often introduce the orthogonality relations using weight functions or considering orthogonality conditions via an extension to measures. Manifestly, on this question, our points of view diverge, because in all our works we consider the orthogonality w.r.t. different linear functionals not necessarily regular. For more details about the multiple orthogonal polynomials, we refer to [5-9], [10, Chapter 23] and the references contained therein.

Definition 1.3 [1,2]: $A$ PS $\left\{P_{n}\right\}_{n \geqslant 0}$ is said to be d-orthogonal polynomials sequence (d-OPS in short) with respect to the $d$-dimensional functional $\mathbf{U}={ }^{t}\left(u_{0}, u_{1}, \ldots, u_{d-1}\right) \in\left(\mathscr{P}^{\prime}\right)^{d}$ if it fulfills the following orthogonality conditions

$$
\begin{aligned}
\left\langle u_{k}, P_{n} P_{m}\right\rangle & =0, m \geqslant d n+k+1, \\
\left\langle u_{k}, P_{n} P_{d n+k}\right\rangle & \neq 0, n \geqslant 0,
\end{aligned}
$$

where $d$ is a positive integer, $k=0,1, \ldots, d-1$, and $n \in \mathbb{N}=\{0,1,2, \ldots\}$.

One of the most important characterization of the $d$-orthogonal polynomials is the fact that they satisfy a standard recurrence relation of order $d+1$ which we write as follows

$$
P_{n+d+1}(x)=\left(x-\beta_{n+d}\right) P_{n+d}(x)-\sum_{\nu=0}^{d-1} \gamma_{n+d-\nu}^{d-1-\nu} P_{n+d-1-\nu}(x), n \geqslant 0,
$$

\footnotetext{
${ }^{1}$ Let $n \in \mathbb{N}$ and write it as $n=m d+j$, with $0 \leqslant j<d$. The nearly diagonal multi-index $\ell(n)$ corresponding to $n$ is then given by $\ell(n)=(m+1, m+1, \ldots, m+1, m, m, \ldots, m)$, with $j$ times $m+1$ and $d-j$ times $m$. If we denote the corresponding multiple orthogonal polynomials by $P_{n}(x)=P_{\ell(n)}(x)$; then the sequence $\left\{P_{n}\right\}_{n \geqslant 0}$ satisfies the $(d+1)$-order recurrence relation (1.7) with the initial conditions (1.8)-(1.9).
} 
with the regularity conditions $\gamma_{n+1}^{0} \neq 0, n \geqslant 0$, and the initial conditions

$$
\begin{aligned}
& P_{0}(x)=1, P_{1}(x)=x-\beta_{0} \text { and if } d \geqslant 2: \\
& P_{n}(x)=\left(x-\beta_{n-1}\right) P_{n-1}(x)-\sum_{\nu=0}^{n-2} \gamma_{n-1-\nu}^{d-1-\nu} P_{n-2-\nu}(x), 2 \leqslant n \leqslant d .
\end{aligned}
$$

When $d=1$, we recover the ordinary orthogonality described above.

The recurrence relation (1.7), with its initial conditions (1.8)-(1.9), plays a primordial role in various works published before, since this is a starting point to provide some explicit examples of $d$-orthogonal polynomials as an extension of the classical orthogonal polynomials.

Definition 1.4 [1]: The d-dimensional functional $\mathbf{U}={ }^{t}\left(u_{0}, u_{1}, \ldots, u_{d-1}\right)$ is called regular if there exists a PS $\left\{P_{n}\right\}_{n \geqslant 0}$ satisfying the conditions (1.5) and (1.6).

In order to provide another criterion equivalent to the regularity conditions stated in the above definition, let us denote by $\left(u_{k}\right)_{n}=\left\langle u_{k}, x^{n}\right\rangle$, the $n$-th moment of the linear functional $u_{k}$ for a fixed $k \in\{0, \ldots, d-1\}$. By $\Delta_{n}^{k}=\operatorname{det}\left(\left(u_{k}\right)_{i+j}\right)_{i, j=0}^{n}, n \geqslant 0$, we denote the ordinary Hankel determinants constructed from the moments of $u_{k}$. It was established in [1, Lemme 2.1] or in [2, p.142] that the conditions which ensure both the regularity of the $d$-dimensional vector $\mathbf{U}$ and, consequently, the existence and uniqueness of the corresponding $d$-OPS are related to generalized Hankel determinants, constructed from the moments of the $d$ linear functionals $u_{0}, u_{1}, \ldots, u_{d-1}$. Such determinants must be non-zero, and hence one has conditions that are analogous, and generalize, those of Hankel's determinants in the ordinary orthogonality. It is to be noted in passing that this condition is often difficult to verify.

Definition 1.5 [11]: The d-dimensional functional $\mathbf{U}={ }^{t}\left(u_{0}, u_{1}, \ldots, u_{d-1}\right)$ is called d-symmetric if the moments of each component $u_{k}, k=0,1, \ldots, d-1$, satisfy

$$
\left(u_{k}\right)_{(d+1) n+j}=\left\langle u_{k}, x^{(d+1) n+j}\right\rangle=0, j=0,1, \ldots, d, n \geqslant 0, \text { for } j \neq k .
$$

Evidently, when $d=1$, the vector $\mathbf{U}$ reduces to $u_{0}$ and the conditions (1.10) lead to the fact that all the odd moments of the symmetric functional $u_{0}$ are zero [4]: $\left(u_{0}\right)_{2 n+1}=\left\langle u_{0}, x^{2 n+1}\right\rangle=0, n \geqslant 0$.

\section{Remarks 1.2:}

(a) By applying the definition of the dual sequence, it is easily seen that, for each $k \in\{0, \ldots, d-1\}$, the moments of the functionals $u_{k}$ satisfy: $\left(u_{0}\right)_{0}=1$ and $\left(u_{k}\right)_{0}=0$ for $k \neq 0$. Then, in accordance with Remarks 1.1 (c), none of the linear functionals $u_{k}, k=1,2, \ldots, d-1$, is regular. Only the first functional $u_{0}$ could be regular and one can associate with it an OPS. Of course, this holds if the regularity conditions described in Definition 1.1. are fulfilled to guarantee the existence and uniqueness of a such OPS.

(b) For $d \geqslant 2$, if $\mathbf{U}$ is $d$-symmetric, then $u_{0}$ is also non regular, since at least one of its Hankel's determinants vanish. Indeed, for $n=0$ in $(1.10)$, we get that $\left(u_{0}\right)_{j}=0, j=1, \ldots, d$, yielding

$$
\Delta_{1}^{0}=\left|\begin{array}{ll}
\left(u_{0}\right)_{0} & \left(u_{0}\right)_{1} \\
\left(u_{0}\right)_{1} & \left(u_{0}\right)_{2}
\end{array}\right|=\left|\begin{array}{ll}
1 & 0 \\
0 & 0
\end{array}\right|=0,
$$


where $\Delta_{n}^{0}, n \geqslant 0$, are the Hankel determinants generated by the moments of $u_{0}$. But (1.11) is in contradiction with the regularity conditions already mentioned in Definition 1.1 (or in Th. 1.2). Hence there is no OPS associated to $u_{0}$. Notice that, in this case, none of the components is regular although the $d$-dimensional functional $\mathbf{U}$ is still regular as required by Definition 1.3.

On account of the above remark, we will give at the end of this part, when $d=2$, some interesting families of classical 2-OPS w.r.t. the regular vector functional $\mathbf{U}={ }^{t}\left(u_{0}, u_{1}\right)$, without either of the two functionals $u_{0}$ and $u_{1}$ being regular. Likewise, we will provide three subcases when the first linear functional $u_{0}$ is a classical one: Laguerre or Hermite. Accordingly, one could argue that we are in a position to establish a connection between the ordinary orthogonality and the two-orthogonality.

Definition 1.6 [11]: A PS $\left\{P_{n}\right\}_{n \geqslant 0}$ is called d-symmetric if each polynomial verifies

$$
P_{n}\left(\omega_{k} x\right)=\omega_{k}^{n} P_{n}(x), n \geqslant 0,
$$

where $\omega_{k}=\exp (2 k i \pi /(d+1))$, for $k=1,2, \ldots, d$, and $\omega_{k}^{d+1}=1$ (these are the $(d+1)$ th roots of unity).

Notice that, if $d=1$, then the identity (1.12) reduces to the well-known identity of a symmetric polynomials.

Theorem 1.7 [11, Th. 4.1]: For every PS $\left\{P_{n}\right\}_{n \geqslant 0} d$-orthogonal with respect to the vector functional $\mathbf{U}={ }^{t}\left(u_{0}, u_{1}, \ldots, u_{d-1}\right)$, the following statements are equivalent.

(1) The d-dimensional functional $\mathbf{U}$ is d-symmetric.

(2) The sequence $\left\{P_{n}\right\}_{n \geqslant 0}$ is d-symmetric.

(3) The sequence $\left\{P_{n}\right\}_{n \geqslant 0}$ satisfies the recurrence relation

$$
P_{n+d+1}(x)=x P_{n+d}(x)-\gamma_{n+1} P_{n}(x), n \geqslant 0,
$$

with $P_{n}(x)=x^{n}$ for $0 \leqslant n \leqslant d$, and $\gamma_{n+1} \neq 0, n \geqslant 0$.

An immediate consequence of this theorem is that a $d$-OPS is $d$-symmetric if and only if there exist $(d+1)$ sequences of $d$-orthogonal polynomials denoted $\left\{P_{n}^{j}\right\}_{n \geqslant 0}, j=0,1, \ldots, d$, and called the components of $\left\{P_{n}\right\}_{n \geqslant 0}$, such that $P_{(d+1) n+j}(x)=x^{j} P_{n}^{j}\left(x^{d+1}\right)$, for $0 \leqslant j \leqslant d$.

As examples, the particular cases $d=1$ and $d=2$ are worth quoting. We check at once that, when we substitute $d=1$ in (1.12), we recover the ordinary quadratic decomposition of a symmetric OPS [4]. For the case $d=2$, the obtaining results provide the cubic decomposition of the 2-symmetric polynomials $\left\{P_{n}\right\}_{n \geqslant 0}$ whose its components $\left\{P_{n}^{j}\right\}_{n \geqslant 0}(j=0,1,2)$ are given by [11]:

$$
P_{3 n}(x)=P_{n}^{0}\left(x^{3}\right) ; P_{3 n+1}(x)=x P_{n}^{1}\left(x^{3}\right) ; P_{3 n+2}(x)=x^{2} P_{n}^{2}\left(x^{3}\right) .
$$

On account of Theorem 1.7, if $\left\{P_{n}\right\}_{n \geqslant 0}$ is a 2-OPS, then the three components $\left\{P_{n}^{j}\right\}_{n \geqslant 0}$ are also 2-OPS.

Before considering our problem in the next section, let us recall the Hahn property described in [12], which is conjointly satisfied by the classical orthogonal polynomial sequences of Hermite, Laguerre, Jacobi and Bessel: the sequence of their derivatives are also orthogonal polynomial sequences. In our view, the extension of this property to define the classical character in the context of $d$-orthogonality is crucial to give new classes of $d$-OPS. For this purpose, we state the following definition. 
Definition 1.8 : A monic d-orthogonal polynomial sequence $\left\{P_{n}\right\}_{n \geqslant 0}$ is called "classical" if the sequence of its derivatives $\left\{Q_{n}:=P_{n+1}^{\prime} /(n+1)\right\}_{n \geqslant 0}$ is also a d-orthogonal polynomial sequence.

Our motivation to adopt this definition is the fact that it agrees with the Hahn's property. Moreover, its direct application for $d=1$ leads to a non-linear system involving the recurrence coefficients whose solutions provide exhaustively the four families of the classical OPS as it was shown in [11]. The same approach is also applied in many other works when $d \geqslant 2$ (see e.g. [11,13-17]. This provides, for example, the entire description of the $d$-symmetric classical $d$-OPS families made in [13] where the authors revealed that there are exactly $2^{d}$ different cases. In particular, for $d=1$, the obtained results correspond to the two $\left(2^{1}\right)$ families of symmetric classical OPS (Hermite and Gegenbauer), and for $d=2$, there are exactly four $\left(2^{2}\right)$ families of 2-symmetric classical 2-OPS. For more details we refer the reader to $[11,18]$.

The case $d=2$ will be the one of interest to us in all what follows. In fact, our main goal in this case is to find explicitly new solutions to the non-linear system governing the coefficients relating to the third-order recurrence relation. By the way, the obtained polynomials provide a new classical of 2-OPS, and so they bring a small step forward in this perspective. It should be noted that, obtaining the complete solution to such a system with all classes of 2-OPS is currently far from being achieved.

\section{A new class of classical 2-orthogonal polynomials}

To begin, take $d=2$ to keep the notations simple. Let $\left\{P_{n}\right\}_{n \geqslant 0}$ be the 2-OPS w.r.t. the vector functional $\mathbf{U}={ }^{t}\left(u_{0}, u_{1}\right)$. Thus, the PS $\left\{P_{n}\right\}_{n \geqslant 0}$ satisfies the orthogonality relations, valid for $k=0$ and $k=1$ :

$$
\begin{aligned}
\left\langle u_{k}, P_{n} P_{m}\right\rangle & =0, m \geqslant 2 n+k+1, \\
\left\langle u_{k}, P_{n} P_{2 n+k}\right\rangle & \neq 0, n \geqslant 0 .
\end{aligned}
$$

Equivalently, $\left\{P_{n}\right\}_{n \geqslant 0}$ satisfies a third-order recurrence relation which we write in the form

$$
\begin{aligned}
& P_{n+3}(x)=\left(x-\beta_{n+2}\right) P_{n+2}(x)-\alpha_{n+2} P_{n+1}(x)-\gamma_{n+1} P_{n}(x), n \geqslant 0, \\
& P_{0}(x)=1, P_{1}(x)=x-\beta_{0}, P_{2}(x)=\left(x-\beta_{1}\right) P_{1}(x)-\alpha_{1}, \\
& \gamma_{n+1} \neq 0, n \geqslant 0, \text { (regularity conditions). }
\end{aligned}
$$

Our main result here consists in the presentation of a new class of 2-OPS with some of its special cases that deserve particular attention. Certain families of polynomials have already been explored across different studies, the others seem to be new. In each case, we give the explicit formulas of the recurrence coefficients and some of their properties as a differential-recurrence relation and a third-order differential equation. As previously stated, we will not discuss here the integral representations of the corresponding linear functionals.

Let us first remember the sequence of polynomials $\left\{Q_{n}:=(n+1)^{-1} P_{n+1}^{\prime}\right\}_{n \geqslant 0}$ with its associated dual sequence denoted by $\left\{v_{n}\right\}_{n \geqslant 0}$. The latter is being related to $\left\{u_{n}\right\}_{n \geqslant 0}$ via $v_{n}^{\prime}=-(n+1) u_{n+1}, n \geqslant 0$.

In the rest of this section we assume the 2-OPS $\left\{P_{n}\right\}_{n \geqslant 0}$ to be classical. Thus, the PS $\left\{Q_{n}\right\}_{n \geqslant 0}$ is also 2-orthogonal (w.r.t. the vector functional $\left.\mathbf{V}={ }^{t}\left(v_{0}, v_{1}\right)\right)$, and so it satisfies a third-order recurrence 
relation given by

$$
\begin{aligned}
& Q_{n+3}(x)=\left(x-\tilde{\beta}_{n+2}\right) Q_{n+2}(x)-\tilde{\alpha}_{n+2} Q_{n+1}(x)-\tilde{\gamma}_{n+1} Q_{n}(x), n \geqslant 0, \\
& Q_{0}(x)=1, Q_{1}(x)=x-\tilde{\beta}_{0}, Q_{2}(x)=\left(x-\tilde{\beta}_{1}\right) Q_{1}(x)-\tilde{\alpha}_{1}, \\
& \tilde{\gamma}_{n+1} \neq 0, \text { (regularity conditions). }
\end{aligned}
$$

By differentiating (2.3) and using (2.6), we easily obtain that each polynomial $P_{n}$ can be written in terms of $Q_{n}, Q_{n-1}, Q_{n-2}$ and $Q_{n-3}$. Hereafter we write this relation by shifting $n \longrightarrow n+3$ as

$$
\begin{aligned}
P_{n+3}(x)=Q_{n+3}(x) & +(n+3)\left(\beta_{n+3}-\tilde{\beta}_{n+2}\right) Q_{n+2}(x)+\left((n+2) \alpha_{n+3}-(n+3) \tilde{\alpha}_{n+2}\right) Q_{n+1}(x) \\
& +\left((n+1) \gamma_{n+2}-(n+3) \tilde{\gamma}_{n+1}\right) Q_{n}(x), n \geqslant 0
\end{aligned}
$$

with the initial conditions

$$
P_{0}(x)=Q_{0}(x)=1, P_{1}(x)=Q_{1}(x)+\beta_{1}-\tilde{\beta}_{0}, P_{2}(x)=Q_{2}(x)+2\left(\beta_{2}-\tilde{\beta}_{1}\right) Q_{1}(x)+\left(\alpha_{2}-\tilde{\alpha}_{1}\right) .
$$

We can now proceed as in $[11,15]$ to establish the system in question and rewriting it in a reduced form. For this, let us put

$$
\begin{aligned}
& \tilde{\beta}_{n}=\beta_{n+1}+\delta_{n}, n \geqslant 0, \\
& \tilde{\alpha}_{n}=\frac{n}{n+1} \alpha_{n+1} \rho_{n}, n \geqslant 1, \quad\left(\rho_{n} \neq 0\right), \\
& \tilde{\gamma}_{n}=\frac{n}{n+2} \gamma_{n+1} \theta_{n}, n \geqslant 1, \quad\left(\theta_{n} \neq 0\right) .
\end{aligned}
$$

Under the transformations (2.11)-(2.13), the relations (2.9)-(2.10) change into

$$
\begin{aligned}
P_{n+3}(x)=Q_{n+3}(x)-(n+3) \delta_{n+2} Q_{n+2}(x) & +(n+2) \alpha_{n+3}\left(1-\rho_{n+2}\right) Q_{n+1}(x) \\
& +(n+1) \gamma_{n+2}\left(1-\theta_{n+1}\right) Q_{n}(x), n \geqslant 0
\end{aligned}
$$

Substituting (2.3)-(2.4) with (2.6)-(2.7) in (2.14)-(2.15) and making use of (2.11)-(2.13), the desired system follows immediately. In its reduced form this system can be written, for all $n \geqslant 1$, as 


$$
\begin{aligned}
& \beta_{n+1}-\beta_{n}=n \delta_{n-1}-(n+2) \delta_{n} ; \\
& \beta_{1}-\beta_{0}=-2 \delta_{0}, \\
& {\left[(n+3)\left(\rho_{n+1}-1\right)+1\right] \frac{\alpha_{n+2}}{n+2}-\left[n\left(\rho_{n}-1\right)+1\right] \frac{\alpha_{n+1}}{n+1}=\delta_{n}^{2} ;} \\
& \left(3 \rho_{1}-2\right) \alpha_{2}-2 \alpha_{1}=2 \delta_{0}^{2}, \\
& {\left[(n+4)\left(\theta_{n+1}-1\right)+1\right] \frac{\gamma_{n+2}}{n+3}-\left[n\left(\theta_{n}-1\right)+1\right] \frac{\gamma_{n+1}}{n+1}=} \\
& \left\{\left[(n+4)\left(\rho_{n+1}-1\right)+1\right] \delta_{n+1}-\left[n\left(\rho_{n+1}-1\right)+1\right] \delta_{n}\right\} \alpha_{n+2} ; \\
& \left(4 \theta_{1}-3\right) \gamma_{2}-3 \gamma_{1}=3\left[\left(4 \rho_{1}-3\right) \delta_{1}-\delta_{0}\right] \alpha_{2}, \\
& {\left[\left(\rho_{n+1}-2\right) \rho_{n}+1\right] \alpha_{n+2} \alpha_{n+1}=} \\
& -\left\{\left[(n+4)\left(\theta_{n}-1\right)+1\right] \delta_{n+1}+\left[\theta_{n}-1\right] \delta_{n}-\left[(n-1)\left(\theta_{n}-1\right)+1\right] \delta_{n-1}\right\} \gamma_{n+1}, \\
& {\left[\left(\rho_{n+2}-2\right) \theta_{n}+1\right] \alpha_{n+3} \gamma_{n+1}+\left[\left(\theta_{n+1}-2\right) \rho_{n}+1\right] \alpha_{n+1} \gamma_{n+2}=0,} \\
& \theta_{n+2}+\frac{1}{\theta_{n}}=2 .
\end{aligned}
$$

Starting with the observation that Equation (2.21) stands out from the rest, since it plays a primordial role to determine all other coefficients. In fact, this is a simple Riccati equation all of whose solutions are given by
A) $\theta_{n}=1, n \geqslant 1$,
В) $\theta_{2 n}=\frac{n+\mu+1}{n+\mu}, \quad \theta_{2 n-1}=1, n \geqslant 1$,
C) $\theta_{2 n}=1$,
$\theta_{2 n-1}=\frac{n+\nu+1}{n+\nu}, n \geqslant 1$,
(where $\mu, \nu \neq-1,-2, \ldots$ ).
D) $\theta_{2 n}=\frac{n+\mu+1}{n+\mu}, \quad \theta_{2 n-1}=\frac{n+\nu+1}{n+\nu}, n \geqslant 1$,

The system under consideration is at present far from being fully solved. Thus to look for other new solutions, we have to work under certain assumptions on the coefficients since this strategy was already generated some families of classical 2-OPS in various previous works (see e.g., [6, 15, 19-21]). For this, observe that the expression in braces in the right-hand side of Equation (2.19) vanishes if and only if $\alpha_{n}=0, n \geqslant 1$, or $\left(\rho_{n+1}-2\right) \rho_{n}+1=0, n \geqslant 1$. The first condition implies that $\beta_{n}=0, n \geqslant 0$. This is easily verified when substituting $\alpha_{n}=0, n \geqslant 1$, in Equations (2.17)-(2.17)'. Under these specific conditions one gets a simple system that was integrally solved by the authors in [11] obtaining the four families of 2-symmetric 2-OPS. Recall that these polynomials are recently reviewed in [18].

When $\alpha_{n} \neq 0, n \geqslant 1$, and $\beta_{n}=0, n \geqslant 0$, the resulting polynomials belong to the Appell's class [11,14]. We certainly believe a priori that Equation (2.19) is the second one to be regarded to progress in the resolution of the above system, since its exploration produce new results as we will see below. For this purpose, we set $\alpha_{n} \neq 0, n \geqslant 1$, and $\beta_{n} \neq 0, n \geqslant 0$. Under these assumptions, we deduce from (2.19) that

$$
\left(\rho_{n+1}-2\right) \rho_{n}+1=0 \Longleftrightarrow\left[(n+4)\left(\theta_{n}-1\right)+1\right] \delta_{n+1}+\left[\theta_{n}-1\right] \delta_{n}-\left[(n-1)\left(\theta_{n}-1\right)+1\right] \delta_{n-1}=0 .
$$


The left-hand side of the equivalence (2.22) is another simple Riccati equation which can be written as

$$
\rho_{n+1}+\frac{1}{\rho_{n}}=2, n \geqslant 1,
$$

whose solutions are explicitly given by

$$
\begin{aligned}
& \text { i) } \rho_{n}=1, n \geqslant 1, \\
& \text { ii) } \left.\rho_{n}=\frac{n+\rho+1}{n+\rho}, n \geqslant 1, \quad \quad \text { (where } \rho \neq-1,-2, \ldots\right) .
\end{aligned}
$$

In the light of the solutions of Equations (2.21)-(2.23), it is clear that various cases may be investigated. However, to provide a new particular solution of the system (2.16)-(2.21), we will mainly focus in this study on the priority case what will referred to as $\mathbf{A}-\mathbf{i}): \theta_{n}=1$ and $\rho_{n}=1, \forall n \geqslant 1$.

This is the straightforward one to be examined, since it is completely resolved as we will see thereafter. Indeed, when $\theta_{n}=\rho_{n}=1$, the right-hand side of the equivalence (2.22) leads to $\delta_{n+1}-\delta_{n-1}=0, n \geqslant 1$. In consequence, the foregoing system reduces to

$$
\begin{aligned}
\beta_{n+1}-\beta_{n} & =n \delta_{n-1}-(n+2) \delta_{n}, n \geqslant 0, \quad\left(\delta_{-1}=0\right), \\
\frac{\alpha_{n+2}}{n+2}-\frac{\alpha_{n+1}}{n+1} & =\delta_{n}^{2}, n \geqslant 0, \\
\frac{\gamma_{n+2}}{n+3}-\frac{\gamma_{n+1}}{n+1} & =\left(\delta_{n+1}-\delta_{n}\right) \alpha_{n+2}, n \geqslant 0, \\
\delta_{n+2}-\delta_{n} & =0, n \geqslant 0 .
\end{aligned}
$$

Our reasoning starts with the observation that $\delta_{n}$ satisfies an homogeneous second-order recurrence relation whose the general solution is given by

$$
\delta_{n}=s+\varepsilon_{n} r,
$$

where $s$ and $r$ are two arbitrary constants (taken here as real parameters), and $\varepsilon_{n}=(-1)^{n}, n \geqslant 0$.

On account of (2.28), it is easy verified that Equations (2.24)-(2.26) give rise to

$$
\begin{aligned}
\beta_{2 n} & =(r-2 s)(2 n)+\beta_{0}, n \geqslant 0, \\
\beta_{2 n+1} & =-(r+2 s)(2 n+1)-r+\beta_{0}, n \geqslant 0 ; \\
\alpha_{2 n+1} & =(2 n+1)\left(\left(r^{2}+s^{2}\right)(2 n)+\alpha_{1}\right), n \geqslant 0 ; \\
\alpha_{2 n+2} & =(2 n+2)\left(\left(r^{2}+s^{2}\right)(2 n+1)+2 r s+\alpha_{1}\right), n \geqslant 0 ; \\
\gamma_{2 n+1} & =(2 n+2)(2 n+1)\left(2 r(r-s)^{2} n+\gamma\right), n \geqslant 0, \\
\gamma_{2 n+2} & =(2 n+3)(2 n+2)\left(-2 r(r+s)^{2}(n+1)-2 r \alpha_{1}+\gamma\right), n \geqslant 0,
\end{aligned}
$$

with $\gamma:=\frac{1}{2} \gamma_{1}$, provided the conditions of regularity remain always fulfilled.

We conclude by observing that the above expressions can be written simply as follows

$$
\begin{aligned}
\beta_{n} & =\left(\varepsilon_{n} r-2 s\right) n-\frac{1}{2}\left(1-\varepsilon_{n}\right) r+\beta_{0}, n \geqslant 0 ; \\
\alpha_{n+1} & =(n+1)\left[\left(r^{2}+s^{2}\right) n+\left(1-\varepsilon_{n}\right) r s+\alpha_{1}\right], n \geqslant 0 ; \\
\gamma_{n+1} & =(n+2)(n+1)\left[\varepsilon_{n}\left(r-\varepsilon_{n} s\right)^{2} r n-\frac{1}{2}\left(1-\varepsilon_{n}\right)\left((r+s)^{2}+2 \alpha_{1}\right) r+\gamma\right], n \geqslant 0 .
\end{aligned}
$$


In addition, substituting (2.29)-(2.31) into (2.11)-(2.13) yields the recurrence coefficients for $\left\{Q_{n}\right\}_{n \geqslant 0}$

$$
\begin{aligned}
& \tilde{\beta}_{n}=\left(s-\varepsilon_{n} r\right) n-\frac{1}{2}\left(1+\varepsilon_{n}\right) r+2 s+\beta_{0}, n \geqslant 0 \\
& \tilde{\alpha}_{n}=n\left[\left(r^{2}+s^{2}\right) n+\left(1-\varepsilon_{n}\right) r s+\alpha_{1}\right], n \geqslant 1 \\
& \tilde{\gamma}_{n}=(n+1) n\left[\varepsilon_{n}\left(r-\varepsilon_{n} s\right)^{2} r n-\frac{1}{2}\left(1-\varepsilon_{n}\right)\left((r+s)^{2}+2 \alpha_{1}\right) r+\gamma\right], n \geqslant 1 .
\end{aligned}
$$

Likewise, when $\theta_{n}=1, \rho_{n}=1$ and $\delta_{n}=s+r \varepsilon_{n}$, the relations (2.14) and (2.15) lead to

$$
P_{n}(x)=Q_{n}(x)-\delta_{n+1} n Q_{n-1}(x), n \geqslant 0 ;\left(Q_{-1}=0\right) .
$$

The relation (2.35) (referred to as the second structure relation) plays an important role in different steps of the present paper as well as throughout some other articles on this topic. This is the key to constructing the above system as previously noted, and then for establishing certain properties for all its solutions. For instance, this allows us to derive the differential-recurrence relation (the main structure relation) and the third-order differential equation satisfied by the resulting polynomials as we will see in the next section. Mention also that, when dealing with the integral representations, the use of (2.35) in determining the distributional differential equations involving $u_{0}$ and $u_{1}$ is of fundamental importance.

\section{Some properties of the polynomials $P_{n}, n=0,1,2, \ldots$}

\subsection{A differential-recurrence relation}

Proposition 3.1 : The 2-OPS $\left\{P_{n}\right\}_{n \geqslant 0}$ defined by the recurrence coefficients (2.29)-(2.31) fulfills the following differential-recurrence relation (first structure relation)

$$
\phi(x) Q_{n}(x)=\left(r^{2}-s^{2}\right) P_{n+1}(x)+a_{n} P_{n}(x)+b_{n} P_{n-1}(x), n \geqslant 0,\left(P_{-1}=0\right),
$$

where

$$
\begin{aligned}
\phi(x) & =\left(r^{2}-s^{2}\right)\left(x-\beta_{0}\right)+(s-r) \alpha_{1}+\gamma \\
a_{n} & =\left(s^{2}-r^{2}\right)\left(s n+\frac{1}{2}\left(1-\varepsilon_{n}\right) r\right)+(s-r) \alpha_{1}+\gamma \\
b_{n} & =n\left[r\left(r^{2}-s^{2}\right)\left(r+\varepsilon_{n} s\right)\left(n-\frac{1}{2}\left(1-\varepsilon_{n}\right)\right)+\left(\varepsilon_{n} r-s\right)\left(\left(1+\varepsilon_{n}\right) r \alpha_{1}-\gamma\right)\right] .
\end{aligned}
$$

Proof: First, since $\delta_{n}=s+r \varepsilon_{n}$ for each $n$, we then have $\delta_{n} \delta_{n+1}=\delta_{0} \delta_{1}=s^{2}-r^{2}$. Now, write (2.35) as

$$
P_{n+1}^{\prime}(x)=(n+1) P_{n}(x)+(n+1) \delta_{n+1} P_{n}^{\prime}(x), n \geqslant 0,
$$

which successively gives for the subscripts $n+2$ and $n+3$ :

$$
\begin{aligned}
& P_{n+2}^{\prime}(x)=(n+2) P_{n+1}(x)+(n+2) \delta_{n} P_{n+1}^{\prime}(x), n \geqslant 0, \\
& P_{n+3}^{\prime}(x)=(n+3) P_{n+2}(x)+(n+3) \delta_{n+1} P_{n+2}^{\prime}(x), n \geqslant 0 .
\end{aligned}
$$

Substituting for $P_{n+2}^{\prime}$ from (3.6) into (3.7), yields

$$
P_{n+3}^{\prime}(x)=(n+3) P_{n+2}(x)+(n+3)(n+2) \delta_{n+1} P_{n+1}(x)+\left(s^{2}-r^{2}\right)(n+3)(n+2) P_{n+1}^{\prime}(x), n \geqslant 0 .
$$


Now, using again (3.5) to write $P_{n}^{\prime}$ as

$$
P_{n}^{\prime}(x)=\frac{1}{(n+1) \delta_{n+1}} P_{n+1}^{\prime}(x)-\frac{1}{\delta_{n+1}} P_{n}(x), n \geqslant 0 .
$$

Moreover, the differentiation of the third-order recurrence relation (2.3) gives

$$
P_{n+2}(x)=P_{n+3}^{\prime}(x)+\alpha_{n+2} P_{n+1}^{\prime}(x)+\gamma_{n+1} P_{n}^{\prime}(x)-\left(x-\beta_{n+2}\right) P_{n+2}^{\prime}(x), n \geqslant 0 .
$$

Consequently, substituting for $P_{n+3}^{\prime}, P_{n+2}^{\prime}$ and $P_{n}^{\prime}$ from (3.8), (3.6), and (3.9), respectively, into (3.10) and using again the recurrence relation (2.3), we obtain

$$
\begin{aligned}
& {\left[\left(r^{2}-s^{2}\right)\left(x-\beta_{n+2}-(n+3) \delta_{n+1}\right)+\frac{\delta_{n+1} \alpha_{n+2}}{n+2}+\frac{\gamma_{n+1}}{(n+1)(n+2)}\right] P_{n+1}^{\prime}(x)=} \\
& \quad\left(r^{2}-s^{2}\right)(n+1) P_{n+1}(x)+\left(\delta_{n+1} \alpha_{n+1}+\frac{\gamma_{n+1}}{n+2}\right) P_{n}(x)+\delta_{n+1} \gamma_{n} P_{n-1}(x), n \geqslant 0 .
\end{aligned}
$$

Lastly, by dividing both sides of (3.11) by $n+1$, the differential-recurrence relation (3.1) follows immediately, with

$$
\begin{aligned}
\phi(x) & =\left(r^{2}-s^{2}\right)\left(x-\beta_{n+2}-(n+3) \delta_{n+1}\right)+\frac{\delta_{n+1} \alpha_{n+2}}{n+2}+\frac{\gamma_{n+1}}{(n+1)(n+2)} \\
a_{n} & =\frac{\delta_{n+1} \alpha_{n+1}}{n+1}+\frac{\gamma_{n+1}}{(n+1)(n+2)} \text { and } b_{n}=\frac{\delta_{n+1} \gamma_{n}}{n+1} .
\end{aligned}
$$

Substituting the coefficients $\beta_{n}, \alpha_{n}$ and $\gamma_{n}$ from (2.29)-(2.31) into the last identities, we find the explicit expressions of the polynomial $\phi$ and the coefficients $a_{n}$ and $b_{n}$ given by (3.2)-(3.4).

\subsection{A third-order differential equation}

Proposition 3.2 : Each polynomial $P_{n}$ of the 2-OPS fulfilling (3.1) satisfies a third-order differential equation of the form

$$
\phi(x) y^{\prime \prime \prime}+p(x) y^{\prime \prime}+q(x, n) y^{\prime}+n y=0, n \geqslant 0,
$$

where

$$
\begin{aligned}
& \phi(x)=\left(r^{2}-s^{2}\right)\left(x-\beta_{0}\right)+(s-r) \alpha_{1}+\gamma ; \\
& p(x)=-2 s\left(x-\beta_{0}\right)+r^{2}-s^{2}+\alpha_{1}, \\
& q(x, n)=-\left(x-\beta_{0}\right)+s(n-1)-\frac{1}{2} r\left(1+\varepsilon_{n}\right) .
\end{aligned}
$$

Proof: Our proof starts from the differential-recurrence (3.1) written in the form

$$
\phi(x) P_{n+1}^{\prime}(x)=\left(r^{2}-s^{2}\right)(n+1) P_{n+1}(x)+(n+1) a_{n} P_{n}(x)+(n+1) b_{n} P_{n-1}(x), n \geqslant 0,
$$

Differentiating twice, we successively obtain

$$
\begin{aligned}
& \phi(x) P_{n+1}^{\prime \prime}(x)=\left(r^{2}-s^{2}\right) n P_{n+1}^{\prime}(x)+(n+1) a_{n} P_{n}^{\prime}(x)+(n+1) b_{n} P_{n-1}^{\prime}(x), n \geqslant 0, \\
& \phi(x) P_{n+1}^{\prime \prime \prime}(x)=\left(r^{2}-s^{2}\right)(n-1) P_{n+1}^{\prime \prime}(x)+(n+1) a_{n} P_{n}^{\prime \prime}(x)+(n+1) b_{n} P_{n-1}^{\prime \prime}(x), n \geqslant 0 .
\end{aligned}
$$


The procedure is to eliminate $P_{n}^{\prime \prime}$ and $P_{n-1}^{\prime \prime}$ in (3.14) and replace them in terms of $P_{n+1}$ and its derivatives. For this, we use the recurrence relation (2.3) which we write after shifting $n \longrightarrow n-1$ as

$$
\gamma_{n} P_{n-1}(x)=\left(x-\beta_{n+1}\right) P_{n+1}(x)-\alpha_{n+1} P_{n}(x)-P_{n+2}(x),\left(P_{-1}=0\right) .
$$

By differentiating, the last equation gives

$$
\gamma_{n} P_{n-1}^{\prime}(x)=\left(x-\beta_{n+1}\right) P_{n+1}^{\prime}(x)-\alpha_{n+1} P_{n}^{\prime}(x)-P_{n+2}^{\prime}(x)+P_{n+1}(x) .
$$

Using (3.6) to eliminate $P_{n+2}^{\prime}$, yields

$$
\gamma_{n} P_{n-1}^{\prime}(x)=\left(x-\beta_{n+1}-(n+2) \delta_{n}\right) P_{n+1}^{\prime}(x)-\alpha_{n+1} P_{n}^{\prime}(x)-(n+1) P_{n+1}(x) .
$$

Differentiating again (3.16) leads to

$$
\gamma_{n} P_{n-1}^{\prime \prime}(x)=\left(x-\beta_{n+1}-(n+2) \delta_{n}\right) P_{n+1}^{\prime \prime}(x)-n P_{n+1}^{\prime}(x)-\alpha_{n+1} P_{n}^{\prime \prime}(x) .
$$

For $n \longrightarrow n+1,(3.17)$ becomes

$$
\gamma_{n+1} P_{n}^{\prime \prime}(x)=\left(x-\beta_{n+2}-(n+3) \delta_{n+1}\right) P_{n+2}^{\prime \prime}(x)-(n+1) P_{n+2}^{\prime}(x)-\alpha_{n+2} P_{n+1}^{\prime \prime}(x) .
$$

Using again (3.6) to eliminate $P_{n+2}^{\prime \prime}$ and $P_{n+2}^{\prime}$ in (3.18), yields

$$
\frac{\gamma_{n+1}}{n+2} P_{n}^{\prime \prime}(x)=f_{n+1}(x) P_{n+1}^{\prime \prime}(x)+g_{n+1}(x) P_{n+1}^{\prime}(x)-(n+1) P_{n+1}(x),
$$

with

$$
f_{n+1}(x)=\delta_{n}\left[x-\beta_{n+2}-(n+3) \delta_{n+1}\right]-\frac{\alpha_{n+2}}{n+2} \text { and } g_{n+1}(x)=x-\beta_{n+2}-(n+3) \delta_{n+1}-(n+1) \delta_{n} .
$$

In the same manner we substitute (3.19) in (3.17) to eliminate $P_{n}^{\prime \prime}$, yielding

$$
\gamma_{n} P_{n-1}^{\prime \prime}(x)=h_{n+1}(x) P_{n+1}^{\prime \prime}(x)+k_{n+1}(x) P_{n+1}^{\prime}(x)+l_{n+1} P_{n+1}(x),
$$

where

$$
\begin{aligned}
& h_{n+1}(x)=x-\beta_{n+1}-(n+2) \delta_{n}-(n+2) \frac{\alpha_{n+1}}{\gamma_{n+1}} f_{n+1}(x), \\
& k_{n+1}(x)=-n-(n+2) \frac{\alpha_{n+1}}{\gamma_{n+1}} g_{n+1}(x) \text { and } l_{n+1}=(n+2)(n+1) \frac{\alpha_{n+1}}{\gamma_{n+1}} .
\end{aligned}
$$

Besides, using (3.19) and (3.20) to eliminate $P_{n}^{\prime \prime}$ and $P_{n-1}^{\prime \prime}$ in (3.14), we obtain

$$
\begin{aligned}
\phi P_{n+1}^{\prime \prime \prime}+\left(s^{2}-r^{2}\right)(n-1) P_{n+1}^{\prime \prime} & -\frac{n+1}{\gamma_{n}} b_{n}\left\{h_{n+1} P_{n+1}^{\prime \prime}+k_{n+1} P_{n+1}^{\prime}+l_{n+1} P_{n+1}\right\} \\
& -\frac{(n+2)(n+1)}{\gamma_{n+1}} a_{n}\left\{f_{n+1} P_{n+1}^{\prime \prime}+g_{n+1} P_{n+1}^{\prime}-(n+1) P_{n+1}\right\}=0 .
\end{aligned}
$$

Remark that $(n+1) b_{n} / \gamma_{n}=\delta_{n+1}$ and $(n+2)(n+1) a_{n} / \gamma_{n+1}=1+(n+2) \delta_{n+1} \alpha_{n+1} / \gamma_{n+1}$, therefore rearranging the terms in the above equation yields

$$
\phi P_{n+1}^{\prime \prime \prime}+\left[\left(s^{2}-r^{2}\right)(2 n+1)-\delta_{n+1}\left(x-\beta_{n+1}\right)-f_{n+1}\right] P_{n+1}^{\prime \prime}+\left[n \delta_{n+1}-g_{n+1}\right] P_{n+1}^{\prime}+(n+1) P_{n+1}=0 .
$$


Replacing both $f_{n+1}$ and $g_{n+1}$ by their expressions in the last differential equation and making use of (2.29)-(2.31), Equation (3.12) follows immediately.

We should note in passing the very important role of the polynomial $\phi$ arising in both (3.1) and (3.12). This appears again as the leading polynomial in the differential equation satisfied by the functional $u_{0}$, which constitutes one of the two equations derived by direct application of the characterization theorem of the classical $d$-OPS [16, Th. 3.1] when $d=2$. Hence, for the 2-OPS highlighted in Section 2, this characterization is specified by the matrix distributional equation $(\boldsymbol{\Phi} \mathbf{U})^{\prime}+\mathbf{\Psi} \mathbf{U}=0$, where $\boldsymbol{\Phi}$ and $\boldsymbol{\Psi}$ are the $2 \times 2$ matrices given by (see $[11,16]$ for more details):

$$
\boldsymbol{\Phi}(x)=\left(\begin{array}{cc}
1 & -\delta_{0} \\
\varphi(x) & \lambda_{0}
\end{array}\right), \boldsymbol{\Psi}(x)=\left(\begin{array}{cc}
0 & 1 \\
\psi(x) & \lambda_{1}
\end{array}\right) \text { and } \mathbf{U}=\left(\begin{array}{l}
u_{0} \\
u_{1}
\end{array}\right),
$$

where $\varphi(x)=-\delta_{1}\left(x-\beta_{0}\right) / \gamma, \psi(x)=\delta_{1}\left(x-\beta_{0}\right) / \gamma, \lambda_{0}=1+\delta_{1} \alpha_{1} / \gamma, \lambda_{1}=-\alpha_{1} / \gamma, \delta_{0}=s+r, \delta_{1}=s-r$.

In addition, a trivial verification shows that $\phi(x)=\gamma|\boldsymbol{\Phi}(x)|$ which reveals the determining role of this polynomial when discussing the integral representations of the two functionals $u_{0}$ and $u_{1}$. For the moment, let us focus on the six subcases that occur naturally when the parameters $\delta_{0}, \delta_{1}$ and $\eta:=2 r \alpha_{1}-\gamma$ take certain specific values. In each case, we recapitulate the first structure relation, the third-order differential equation fulfilled by the 2-OPS $\left\{P_{n}\right\}_{n \geqslant 0}$ and the related recurrence coefficients. All these polynomials still obey the second structure relation (2.35).

\subsection{The special cases}

I. For $\delta_{1}=\delta_{0}=0 \Leftrightarrow s=r=0$ with $\eta=-\gamma \neq 0$, the polynomials $P_{n}, n \geqslant 0$, obey each of the following structure relation and differential equation:

$$
\begin{aligned}
& Q_{n}(x)=P_{n}(x), \\
& \gamma y^{\prime \prime \prime}+\alpha_{1} y^{\prime \prime}-\left(x-\beta_{0}\right) y^{\prime}+n y=0,
\end{aligned}
$$

with the recurrence coefficients $\beta_{n}=\beta_{0}, \alpha_{n+1}=\alpha_{1}(n+1), \gamma_{n+1}=\gamma(n+2)(n+1), n \geqslant 0$.

By virtue of (3.23), it is clear that these polynomials belong to the class of Appell polynomials sharing the basic property $P_{n+1}^{\prime}=(n+1) P_{n}$. They were comprehensively studied in the context of $d$ orthogonality $(d \geqslant 1)$ by the first author in [14]. For $d=1$, we recover the classical Hermite polynomials.

Now, when $d=2$, the following three subcases need to be considered separately.

- Subcase I.1: $\gamma=1, \beta_{0}=0$ and $\alpha_{1}:=2 \alpha \neq 0$ (with $\alpha>0$ ).

- Subcase I.2: $\gamma=1, \beta_{0}=0$ and $\alpha_{1}=0$.

- Subcase I.3: $\gamma=1 / 9, \beta_{0}=0$ and $\alpha_{1}=0$.

Clearly, the Subcase I.1 provides an Appell-type polynomials with the following recurrence coefficients $\beta_{n}=0, \alpha_{n+1}=2 \alpha(n+1), \gamma_{n+1}=(n+2)(n+1)$.

The Subcase I.2 produces the 2-symmetric polynomials referred to as the Airy polynomials in [22] and denoted there by $\mathrm{Pi}_{n}, n \geqslant 0$. In this subcase, we tacitly adopt this notation instead of $P_{n}, n \geqslant 0$. Their recurrence coefficients are $\beta_{n}=0, \alpha_{n+1}=0, \gamma_{n+1}=(n+2)(n+1), n \geqslant 0$. 
Remember that these polynomials are related to the Airy function of the first kind $\operatorname{Ai}(x)$ via the Airy transform of the monomial $x^{n}$ as

$$
\operatorname{Pi}_{n}(x)=x^{n} * \operatorname{Ai}(x)=\int_{-\infty}^{+\infty} t^{n} \operatorname{Ai}(x-t) d t, n \geqslant 0,
$$

where the original Airy function $\operatorname{Ai}(x)$ is defined by the Airy integral

$$
\operatorname{Ai}(x)=\frac{1}{\pi} \int_{0}^{+\infty} \cos \left(x t+\frac{t^{3}}{3}\right) d t, x \geqslant 0
$$

Besides, the polynomials $\mathrm{Pi}_{n}, n \geqslant 0$, satisfy the differential-recurrence formula

$$
\mathrm{Pi}_{n+1}(x)=x \mathrm{Pi}_{n}(x)-\mathrm{Pi}_{n}^{\prime \prime}(x), n \geqslant 0,
$$

which combined with (3.23) leads to the third-order recurrence relation fulfilled by the PS $\left\{\mathrm{Pi}_{n}\right\}_{n \geqslant 0}$. Mention that these polynomials are the first ones investigated in [18] where the authors gave most notably the integral representations of their corresponding linear functionals via the Airy function $\operatorname{Ai}(x)$ and its derivative. Obviously, we will take account of these representations in [23].

Lastly, in Subcase I.3, we encounter the 2 -symmetric polynomials $P_{n}, n=0,1,2, \ldots$, whose the recurrence coefficients are given by $\beta_{n}=0, \alpha_{n+1}=0, \gamma_{n+1}=3^{-2}(n+2)(n+1)$.

By setting $\bar{B}_{n}(x):=a^{-n} B_{n}(a x+b), n \geqslant 0$, for $(a ; b) \in \mathbb{C}^{*} \times \mathbb{C}$ the shifted polynomials for the polynomials $B_{n}, n \geqslant 0$, it is easily verified that the polynomials $P_{n}, n \geqslant 0$, are a shifted Airy polynomials $\mathrm{Pi}_{n}, n \geqslant 0$, (with $a=3^{\frac{2}{3}}$ and $b=0$ ). Accordingly, we get the identities $P_{n}(x)=\overline{\mathrm{P}} \mathrm{i}_{n}(x)=$ $3^{-\frac{2}{3} n} \mathrm{Pi}_{n}\left(3^{\frac{2}{3}} x\right), n \geqslant 0$. For this reason, the notation $\mathrm{Pi}_{n}$ is adopted in Subcase I.2.

Furthermore, by virtue of the symmetry property of the 2-OPS $\left\{P_{n}\right\}_{n \geqslant 0}$, the cubic decomposition described in (1.14) leads to a connection between the three cubic components $P_{n}^{j}, n \geqslant 0, j \in\{0,1,2\}$ (of $\left.P_{n}, n \geqslant 0\right)$ and the hypergeometric polynomials given by $B_{n}^{\alpha, \beta}(x):={ }_{1} F_{2}(-n ; 1+\alpha, 1+\beta ; x), n \geqslant 0$. For a deeper study of these polynomials, we refer to [19] and references therein. This connection is given through the following three relations

$$
P_{n}^{0}(x)=\hat{B}_{n}^{-\frac{2}{3},-\frac{1}{3}}(x) ; P_{n}^{1}(x)=\hat{B}_{n}^{-\frac{1}{3}, \frac{1}{3}}(x) ; P_{n}^{2}(x)=\hat{B}_{n}^{\frac{1}{3}, \frac{2}{3}}(x),
$$

where $\hat{B}_{n}^{\alpha, \beta}=(-1)^{n}(1+\alpha)_{n}(1+\beta)_{n} B_{n}^{\alpha, \beta}, n \geqslant 0$, are the monic polynomials related to $B_{n}^{\alpha, \beta}, n \geqslant 0$. Based on the relations (3.25), a connection between Airy polynomials and the polynomials $\hat{B}_{n}^{\alpha, \beta}, n \geqslant 0$, is then established as follows

$$
\mathrm{Pi}_{3 n}(x)=3^{3 n} \hat{B}_{n}^{-\frac{2}{3},-\frac{1}{3}}\left(3^{-2} x^{3}\right) ; \mathrm{Pi}_{3 n+1}(x)=3^{3 n} x \hat{B}_{n}^{-\frac{1}{3}, \frac{1}{3}}\left(3^{-2} x^{3}\right) ; \mathrm{Pi}_{3 n+2}(x)=3^{3 n} x^{2} \hat{B}_{n}^{\frac{1}{3}, \frac{2}{3}}\left(3^{-2} x^{3}\right) .
$$

Moreover, it was also shown in the same papier that the polynomials $\hat{B}_{n}^{\alpha, \beta}, n \geqslant 0$, are 2-OPS w.r.t. two weight functions associated with Macdonald functions. Recall that an analogous polynomials of the $\hat{B}_{n}^{\alpha, \beta}, n=0,1, \ldots$, are also studied in [21] where the authors considered the orthogonality conditions of these polynomials w.r.t. a system of two positive weight functions associated with Macdonald functions. 
II. For $\delta_{1}=\delta_{0} \neq 0 \Leftrightarrow s \neq 0, r=0$ with $\eta=-\gamma \neq 0$, the polynomials $P_{n}, n \geqslant 0$, satisfy

$$
\begin{aligned}
& \phi(x) Q_{n}(x)=-s^{2} P_{n+1}(x)+a_{n} P_{n}(x)+b_{n} P_{n-1}(x), \\
& \phi(x) y^{\prime \prime \prime}+p(x) y^{\prime \prime}+q(x, n) y^{\prime}+n y=0,
\end{aligned}
$$

where

$$
\begin{aligned}
& \phi(x)=-s^{2}\left(x-\beta_{0}\right)+s \alpha_{1}+\gamma, \quad a_{n}=s^{3} n+s \alpha_{1}+\gamma, \quad b_{n}=s \gamma n, \\
& p(x)=-2 s\left(x-\beta_{0}\right)-s^{2}+\alpha_{1}, \quad q(x, n)=-\left(x-\beta_{0}\right)+s(n-1),
\end{aligned}
$$

with the recurrence coefficients $\beta_{n}=-2 s n+\beta_{0}, \alpha_{n+1}=(n+1)\left(s^{2} n+\alpha_{1}\right), \gamma_{n+1}=\gamma(n+2)(n+1), n \geqslant 0$.

Subcase II.1: Herein we encounter the classical 2-OPS studied by the first author in [15] (denoted there Case $A$ ). In particular, for $s=-1$ and $\gamma=1$, with an appropriate change of the variable, the singularity of the third-order differential equation can be placed at the origin. To do this, we take $\beta_{0}-\alpha_{1}+1=0$. Setting $\alpha_{1}=\alpha+3$, the last equality yields $\beta_{0}=\alpha+2$, and so the recurrence coefficients become $\beta_{n}=2 n+\alpha+2, \alpha_{n+1}=(n+1)(n+\alpha+3), \gamma_{n+1}=(n+2)(n+1)$.

Then, the resulting 2-OPS consists of Laguerre type with one parameter that are 2-orthogonal w.r.t. the pair of weight functions associated with the modified Bessel function of the first kind.

Notice that these polynomials were also studied in [6] where the authors considered the pair of positive weight functions associated with the modified Bessel function of the first kind.

Subcase II.2: As stated above the case $\eta=0$ cannot occur, since this contradicts our assumption.

III. For $\delta_{1}=-\delta_{0} \neq 0 \Leftrightarrow s=0, r \neq 0$, taking into account the parameter $\eta$, two subcases must be distinguished.

- Subcase III.1: $s=0, r \neq 0$ and $\eta \neq 0$.

- Subcase III.2: $s=0, r \neq 0$ and $\eta=0\left(\Leftrightarrow \gamma=2 r \alpha_{1}\right)$.

For the Subcase III.1, we obtain that the polynomials $P_{n}, n \geqslant 0$, fulfill

$$
\begin{aligned}
& \phi(x) Q_{n}(x)=r^{2} P_{n+1}(x)+a_{n} P_{n}(x)+b_{n} P_{n-1}(x), \\
& \phi(x) y^{\prime \prime \prime}+p(x) y^{\prime \prime}+q(x, n) y^{\prime}+n y=0,
\end{aligned}
$$

where $\phi(x)=r^{2}\left(x-\beta_{0}\right)-r \alpha_{1}+\gamma$,

$$
\begin{aligned}
& a_{n}=-\frac{1}{2}\left(1-\varepsilon_{n}\right) r^{3}-r \alpha_{1}+\gamma, b_{n}=r n\left[r^{3}(n-1)+\frac{1}{2}\left(1+\varepsilon_{n}\right)\left(r^{3}+2 r \alpha_{1}\right)-\varepsilon_{n} \gamma\right], \\
& p(x)=r^{2}+\alpha_{1}, q(x, n)=-\left(x-\beta_{0}\right)-\frac{1}{2}\left(1+\varepsilon_{n}\right) r,
\end{aligned}
$$

with the recurrence coefficients:

$$
\begin{aligned}
\beta_{n} & =\varepsilon_{n} r n-\frac{1}{2}\left(1-\varepsilon_{n}\right) r+\beta_{0}, \alpha_{n+1}=(n+1)\left(r^{2} n+\alpha_{1}\right), n \geqslant 0, \\
\gamma_{n+1} & =\varepsilon_{n}(n+2)(n+1)\left[r^{3} n+\frac{1}{2}\left(1-\varepsilon_{n}\right)\left(r^{3}+2 r \alpha_{1}\right)+\varepsilon_{n} \gamma\right], n \geqslant 0 .
\end{aligned}
$$

In such case, taking $r=1$, we recover again the classical 2-OPS obtained in [15] (denoted Case $B$ ). However, the integral representations of their associated linear functionals are not yet given. They will 
be examined in the next study [23]. In order to deal with this question, it is once again convenient to move the singularity of the third-order differential equation to the origin by setting $\beta_{0}+\alpha_{1}-\gamma=0$.

In the Subcase III.2, the polynomials $P_{n}, n \geqslant 0$, satisfy

$$
\begin{aligned}
& \phi(x) Q_{n}(x)=r P_{n+1}(x)+a_{n} P_{n}(x)+b_{n} P_{n-1}(x), \\
& \phi(x) y^{\prime \prime \prime}+p(x) y^{\prime \prime}+q(x, n) y^{\prime}+n y=0,
\end{aligned}
$$

where

$$
\begin{aligned}
& \phi(x)=r\left(x-\beta_{0}\right)+\alpha_{1}, \\
& a_{n}=-\frac{1}{2}\left(1-\varepsilon_{n}\right) r^{2}+\alpha_{1}, \quad b_{n}=r n\left[r^{2} n-\frac{1}{2}\left(1-\varepsilon_{n}\right)\left(r^{2}-2 \alpha_{1}\right)\right], \\
& p(x)=r^{2}+\alpha_{1}, \quad q(x, n)=-\left(x-\beta_{0}\right)-\frac{1}{2}\left(1+\varepsilon_{n}\right) r .
\end{aligned}
$$

The recurrence coefficients are given by

$$
\begin{aligned}
\beta_{n} & =\varepsilon_{n} r n-\frac{1}{2}\left(1-\varepsilon_{n}\right) r+\beta_{0}, \alpha_{n+1}=(n+1)\left(r^{2} n+\alpha_{1}\right), n \geqslant 0, \\
\gamma_{n+1} & =\varepsilon_{n} r(n+2)(n+1)\left[r^{2} n+\frac{1}{2}\left(1-\varepsilon_{n}\right) r^{2}+\left(1+\varepsilon_{n}\right) \alpha_{1}\right], n \geqslant 0 .
\end{aligned}
$$

If we take $r=1$ and $\beta_{0}=\alpha_{1}=\alpha+1$, we get that the linear functional $u_{0}$ satisfies the first order functional equation $\left(\sigma(x) u_{0}\right)^{\prime}+\tau(x) u_{0}=0$, with the two polynomials $\sigma(x)=x$ and $\tau(x)=x-\alpha-1$. This actually shows that $u_{0}$ is the classical linear functional of Laguerre.

IV. For $\delta_{1}=0, \delta_{0} \neq 0 \Leftrightarrow s=r \neq 0$, on account of $\eta$, we distinct the two subcases.

- Subcase IV.1: $s=r \neq 0$ and $\eta \neq 0$.

- Subcase IV.2: $s=r \neq 0$ and $\eta=0$.

For the Subcase IV.1, we get that the polynomials $P_{n}, n \geqslant 0$, satisfy

$$
\begin{aligned}
& Q_{n}(x)=P_{n}(x)+\left(1-\varepsilon_{n}\right) r n P_{n-1}(x), \\
& \gamma y^{\prime \prime \prime}+p(x) y^{\prime \prime}+q(x, n) y^{\prime}+n y=0,
\end{aligned}
$$

where

$$
p(x)=-2 r\left(x-\beta_{0}\right)+\alpha_{1}, \quad q(x, n)=-\left(x-\beta_{0}\right)+r\left[n-\frac{1}{2}\left(3+\varepsilon_{n}\right)\right],
$$

with the recurrence coefficients

$$
\begin{aligned}
\beta_{n} & =-\left(2-\varepsilon_{n}\right) r n-\frac{1}{2}\left(1-\varepsilon_{n}\right) r+\beta_{0}, \alpha_{n+1}=(n+1)\left[2 r^{2} n+\left(1-\varepsilon_{n}\right) r^{2}+\alpha_{1}\right], n \geqslant 0, \\
\gamma_{n+1} & =-(n+2)(n+1)\left[2\left(1-\varepsilon_{n}\right) r^{3}(n+1)+\left(1-\varepsilon_{n}\right) r \alpha_{1}-\gamma\right], n \geqslant 0 .
\end{aligned}
$$

To get the result we need in this subcase, we put $r=1$ and $\gamma=1$, under the condition $\alpha_{1} \neq \frac{1}{2}$.

In the Subcase IV.2, the polynomials $P_{n}, n \geqslant 0$, satisfy

$$
\begin{aligned}
& Q_{n}(x)=P_{n}(x)+\left(1-\varepsilon_{n}\right) r n P_{n-1}(x), \\
& \gamma y^{\prime \prime \prime}+p(x) y^{\prime \prime}+q(x, n) y^{\prime}+n y=0,
\end{aligned}
$$

where

$$
p(x)=-2 r\left(x-\beta_{0}\right)+\alpha_{1}, \quad q(x, n)=-\left(x-\beta_{0}\right)+r\left[n-\frac{1}{2}\left(3+\varepsilon_{n}\right)\right],
$$


with the recurrence coefficients

$$
\begin{aligned}
\beta_{n} & =-\left(2-\varepsilon_{n}\right) r n+\frac{1}{2}\left(1+\varepsilon_{n}\right) r+\beta_{0}, \alpha_{n+1}=(n+1)\left[2 r^{2} n+\left(1-\varepsilon_{n}\right) r^{2}+\alpha_{1}\right], n \geqslant 0, \\
\gamma_{n+1} & =-r(n+2)(n+1)\left[2\left(1-\varepsilon_{n}\right) r^{2}(n+1)-\left(1+\varepsilon_{n}\right) \alpha_{1}\right], n \geqslant 0 .
\end{aligned}
$$

By choosing $\alpha_{1}=1 / 2$ and $\beta_{0}=0$, we get that the linear functional $u_{0}$ satisfies the functional equation $u_{0}^{\prime}+2 x u_{0}=0$. This implies that the functional $u_{0}$ coincides with the classical functional of Hermite.

V. For $\delta_{1} \neq 0, \delta_{0}=0 \Leftrightarrow s=-r \neq 0$, only the subcase relative to $\eta \neq 0$ take place as we will clarify thereafter (cf. Remark 3.1). To this effect, the polynomials $P_{n}, n \geqslant 0$, fulfill

$$
\begin{aligned}
& Q_{n}(x)=P_{n}-\left(1+\varepsilon_{n}\right) r n P_{n-1}, \\
& -\eta y^{\prime \prime \prime}+p(x) y^{\prime \prime}+q(x, n) y^{\prime}+n y=0,
\end{aligned}
$$

where

$$
p(x)=2 r\left(x-\beta_{0}\right)+\alpha_{1}, \quad q(x, n)=-\left(x-\beta_{0}\right)-r\left[n-\frac{1}{2}\left(1-\varepsilon_{n}\right)\right],
$$

with the recurrence coefficients

$$
\begin{aligned}
\beta_{n} & =\left(2+\varepsilon_{n}\right) r n-\frac{1}{2}\left(1-\varepsilon_{n}\right) r+\beta_{0}, \alpha_{n+1}=(n+1)\left[2 r^{2} n-\left(1-\varepsilon_{n}\right) r^{2}+\alpha_{1}\right], n \geqslant 0 \\
\gamma_{n+1} & =(n+2)(n+1)\left[2\left(1+\varepsilon_{n}\right) r^{3} n-\left(1-\varepsilon_{n}\right) r \alpha_{1}+\gamma\right], n \geqslant 0 .
\end{aligned}
$$

Subcase V.1. The subcase to be explored in [23] arise when taking $r=1, \gamma=1$, with $\alpha_{1} \neq \frac{1}{2}$.

\section{Remark 3.1:}

For $s=-r \neq 0$, it is clear that the subcase with $\eta=0$ cannot occur. In fact, when the two restrictions are simultaneously imposed, we get $\gamma_{n+1}=r\left(1+\varepsilon_{n}\right)(n+2)(n+1)\left(2 r^{2} n+\alpha_{1}\right)$, and so $\gamma_{2 n}=0$ for each $n \geqslant 1$, since $\varepsilon_{n}=(-1)^{n}$, which contradicts the regularity conditions $(2.5)$.

VI. For $\delta_{1} \neq 0, \delta_{0} \neq 0 \Leftrightarrow s \neq \pm r$, only the subcase $\eta=0\left(\Leftrightarrow \gamma=2 r \alpha_{1}\right)$ will be considered. Before making use these conditions, let us gather the proprieties of the polynomials $P_{n}, n \geqslant 0$ as follows:

$$
\begin{aligned}
& \phi(x) Q_{n}(x)=-\delta_{0} \delta_{1} P_{n+1}(x)+a_{n} P_{n}(x)+b_{n} P_{n-1}(x), \\
& \phi(x) y^{\prime \prime \prime}+p(x) y^{\prime \prime}+q(x, n) y^{\prime}+n y=0,
\end{aligned}
$$

where $\phi(x)=-\delta_{0} \delta_{1}\left(x-\beta_{0}\right)+\delta_{0} \alpha_{1}$,

$$
\begin{aligned}
& a_{n}=\delta_{0} \delta_{1}\left[s n+\frac{1}{2}\left(1-\varepsilon_{n}\right) r\right]+\delta_{0} \alpha_{1}, \quad b_{n}=n r \delta_{0}\left[\delta_{1}\left(r+\varepsilon_{n} s\right)\left(n-\frac{1}{2}\left(1-\varepsilon_{n}\right)\right)+\left(1-\varepsilon_{n}\right) \alpha_{1}\right], \\
& p(x)=-2 s\left(x-\beta_{0}\right)-\delta_{0} \delta_{1}+\alpha_{1}, \quad q(x, n)=-\left(x-\beta_{0}\right)+s(n-1)-\frac{1}{2} r\left(1+\varepsilon_{n}\right) .
\end{aligned}
$$

Their recurrence coefficients are given by

$$
\begin{aligned}
\beta_{n} & =\left(\varepsilon_{n} r-2 s\right) n-\frac{1}{2}\left(1-\varepsilon_{n}\right) r+\beta_{0}, \quad \alpha_{n+1}=(n+1)\left[\left(r^{2}+s^{2}\right) n+\left(1-\varepsilon_{n}\right) r s+\alpha_{1}\right], n \geqslant 0, \\
\gamma_{n+1} & =(n+2)(n+1)\left[\varepsilon_{n}\left(s-\varepsilon_{n} r\right)^{2}\left(r n+\frac{1}{2}\left(1-\varepsilon_{n}\right)\right)+\left(1+\varepsilon_{n}\right) r \alpha_{1}\right], n \geqslant 0 .
\end{aligned}
$$


- Subcase VI.1. As in Subcase III.2, for $\delta_{1}=-1(\Leftrightarrow s=r-1)$ and $\beta_{0}=\alpha_{1}=\alpha+1$, we find once again that the linear functional $u_{0}$ corresponds to the classical functional of Laguerre, since it satisfies the first order equation $\left(x u_{0}\right)^{\prime}+(x-\alpha-1) u_{0}=0$. The second functional $u_{1}$ satisfies $(2 r-1) u_{1}^{\prime}-u_{1}=u_{0}^{\prime}$, provided that $r \neq 1 / 2$ and $r \neq 0$. Because $r=1 / 2$ implies $s=-1 / 2$, which contradicts our assumptions $s \neq \pm r$, and $r=0$ leads to a contradiction with the regularity conditions. However, it is to be noted that for $r=1$ (so $s=0$ ) we recover the result given in Subcase III.2.

\section{Acknowledgments}

The authors would like to express their gratitude to the anonymous referee for his/her thorough reading of the manuscript which helped us to improve considerably the text, and for pointing out the additional reference [3].

\section{Disclosure statement}

No potential conflict of interest was reported by the authors.

\section{References}

[1] Maroni P. L'orthogonalité et les récurrences de polynômes d'ordre supérieur à deux. Ann Fac Sci Toulouse. 1989;10(1):105-139.

[2] Van Iseghem J. Vector orthogonal relations. Vector QD-algorithm. J Comput Appl Math. 1987;19:141-150.

[3] Maroni P. Une théorie algébrique des polynômes orthogonaux. Application aux polynômes orthogonaux semi-classiques. In: Brezinski C, Gori L, Ronveaux A, editors. IMACS Annals on Computing and Applied Mathematics. Basel: Baltzer; 1991. p. 95-130.(Orthogonal Polynomials and their Applications; 9).

[4] Chihara TS. An introduction to orthogonal polynomials. New York: Gordon and Breach; 1978.

[5] Aptekarev AI. Multiple orthogonal polynomials. J Comput Appl Math. 1998;99:423-447.

[6] Coussement E, Van Assche W. Multiple orthogonal polynomials associated with the modified Bessel functions of the first kind. Construct Approx. 2003;19:237-263.

[7] Coussement E, Van Assche W. Some classical multiple orthogonal polynomials. J Comput Appl Math. 2001;127:317-347.

[8] Kuijlaars ABJ. Multiple orthogonal polynomial ensembles, Recent trends in orthogonal polynomials and approximation theory. Contemporary Mathematics, Vol. 507. Providence, RI: American Mathematical Society; 2010. p. 155-176.

[9] Nikishin EM, Sorokin VN. Rational approximations and orthogonality, Translations of Mathematical Monographs, Vol. 92. Providence, RI: American Mathematical Society; 1991.

[10] Ismail MEH. Classical and quantum orthogonal polynomials in one variable. Encyclopedia of Mathematics and its Applications, Vol. 98. Cambridge: Cambridge University Press; 2005. 
[11] Douak K, Maroni P. Les polynômes orthogonaux "classiques" de dimension deux. Analysis. 1992;12:71-107.

[12] Hahn W. Über die Jacobischen polynome und zwei verwandte polynomklassen. Math Zeit. 1935;39:634-638.

[13] Ben Cheikh Y, Douak K. On the classical $d$-orthogonal polynomials defined by certain generating functions, I. Bull Belg Math Soc. 2000;7:107-124.

[14] Douak K. The relation of the $d$-orthogonal polynomials to the Appell polynomials. J Comput Appl Math. 1996;70:279-295.

[15] Douak K. On 2-orthogonal polynomials of Laguerre type. Internat J of Math and Math Sci. 1999;22(1):29-48.

[16] Douak K, Maroni P. Une caractérisation des polynômes $d$-orthogonaux "classiques". J Approx Theory. 1995;82:177-204.

[17] Maroni P, Mesquita TA. Two-orthogonal polynomial sequences as eigenfunctions of a third-order differential operator. Medit J Math. 2016;13(2):687-701.

[18] Loureiro AF, Van Assche W. Threefold symmetric Hahn-classical multiple orthogonal polynomials. Anal Appl, to appear. DOI:10.1142/S0219530519500106.

[19] Ben Cheikh Y, Douak K. On two-orthogonal polynomials related of the Bateman $J_{n}^{u, v}$-function. Methods and Appl of Analysis. 2000;7(4):641-662.

[20] Maroni P. Two-dimensional orthogonal polynomials, their associated sets and the co-recursive sets. Numerical Algorithms. 1992;3:299-312.

[21] Van Assche W, Yakubovich SB. Multiple orthogonal polynomials associated with Macdonald functions. Integral Transforms Spec Funct. 2000;9:229-244.

[22] Soares M, Vallée O. Airy functions and applications to physics. London: Imperial College Press; 2004.

[23] Douak K, Maroni P. On a new class of 2-orthogonal polynomials, II: The integral representations. In preparation. 